\title{
Artigo Original / Original Paper \\ Ampliação do conhecimento biogeográfico de Pleurophora pulchra (Lythraceae) com enfoque em biologia da conservação
}

Increasing biogeographical knowledge of Pleurophora pulchra (Lythraceae) with conservation biology approach

\author{
Ramon Athayde de Souza Cavalcante ${ }^{1}$, Fellipe Alves Ozorio do Nascimento ${ }^{1}$, \\ Mary Ann Saraiva Bezerra Fornelos Pereira ${ }^{2}$, Dúlio Paulino de Souza ${ }^{1}$, Andre Paviotti Fontana ${ }^{1}$, \\ Vinícius Messas Cotarelli ${ }^{1}$, Marcondes Albuquerque de Oliveira ${ }^{3}$ \& Edson Gomes de Moura Júnior ${ }^{1,4}$
}

\begin{abstract}
Resumo
Descrita no ano de 2015, o conhecimento da biogeografia de Pleurophora pulchra é limitado, fato que impossibilitou sua inclusão na primeira avaliação sobre Áreas Prioritárias para Conservação da Flora do Domínio Caatinga (APCFCs) e Unidades de Conservação (UCs). Nesse contexto, propusemos descrever o potencial biogeográfico de P. pulchra na Caatinga através de modelagem de distribuição de espécies (MDE), visando ampliar as discussões sobre APCFCs e UCs. Utilizamos o algoritmo Maxent para computar o modelo final (MDEf), e o teste Binomial para avaliar o desempenho dos mesmos. Constatamos que $P$. pulchra está ajustada a ambientes de elevada aridez, com baixa precipitação anual, grande sazonalidade na precipitação, temperaturas estáveis nos meses mais frios e solos pouco espessos, variando de imperfeitamente à bem drenados. O MDEf revelou áreas com adequabilidade ambiental predominantemente na depressão sertaneja meridional da Bahia, Pernambuco e Paraíba, além de áreas do planalto da Borborema da Paraíba. As UCs APAs Cariri e Lago de Sobradinho, APCFCs Jatobá e Cariri Paraibano se destacaram pela representatividade da área com adequabilidade ambiental superior a 0.5. O resultado do teste binomial indicou bom desempenho de $\operatorname{MDEf}(\mathrm{p}<0.001 \mathrm{t}=1)$ e, portanto, reforçou a possibilidade de novos registros de $P$. pulchra nas áreas supracitadas.
\end{abstract}

Palavras-chave: ameaça de extinção, biologia da conservação, MDE.

\begin{abstract}
Having been recently described (2015), knowledge of the biogeography of Pleurophora pulchra is limited, which made its inclusion in the first evaluation of Priority Areas for Conservation of the Flora of the Caatinga Domain (PACFCs) and Conservation Units (CUs) impossible. We describe the biogeographical potential of $P$. pulchra in the Caatinga using species distribution modeling (SDM), with the aim of broadening discussions about PACFCs and CUs. We used the Maxent algorithm to compute the final model (SDMf), and the Binomial test to evaluate their performance. We found that P. pulchra tends to occur in environments with high aridity, low annual precipitation, intense precipitation seasonality, stable temperatures in the colder months, and thin soils that range from poorly to well drained. The SDMf revealed areas with environmental suitability predominantly in the Southern Sertaneja Depression of the states of Bahia, Pernambuco and Paraíba, as well as areas of the Borborema Plateau in Paraíba. The CUs APAs Cariri and Lago de Sobradinho, and the PACFCs Jatobá and Cariri Paraibano stood out as having environmental suitability values above 0.5 . The binomial test exhibited good performance for SDMf $(\mathrm{p}<0.001 \mathrm{t}=1)$, reinforcing the possibility of new records of $P$. pulchra in the aforementioned areas. Key words: extinction threat, conservation biology, SDM.
\end{abstract}

\footnotetext{
${ }^{1}$ Universidade Federal do Vale do São Francisco (UNIVASF), Núcleo de Ecologia e Monitoramento Ambiental (NEMA), BR-407, km 12, lote 543, Projeto de Irrigação Nilo Coelho s/n, 56300-000, Petrolina, PE, Brasil.

${ }^{2}$ Instituto Federal do Sertão Pernambucano (IF Sertão), R. Aristarco Lopes 240, Centro, 56302-100, Petrolina, PE, Brasil.

${ }^{3}$ Instituto Agronômico de Pernambuco (IPA), Av. General San Martin 1371, Bongi, 50761-000, Recife, PE, Brasil.

${ }^{4}$ Autor para correspondência: mourajunioreg@gmail.com
} 


\section{Introdução}

Rico em biodiversidade, o domínio fitogeográfico da Caatinga (DFC), assim como outras regiões brasileiras, vem sofrendo com a ação do homem através da supressão de sua vegetação para implantação de áreas agricultáveis (Castelletti et al. 2004), tornando os solos pobres e inférteis, com tendências à desertificação e perda de biodiversidade (Sá et al. 2010). Ao mesmo tempo, o baixo percentual $7.58 \%$ de áreas do DFC representadas em unidades de conservação (UCs) (MMA 2016b) tornam os estudos que categorizam essas unidades ou áreas prioritárias para conservação da flora do domínio Caatinga (APCFCs) imprescindíveis no contexto de conservação da biodiversidade desse domínio (Tabarelli \& Silva 2002).

O uso de sistemas de informações geográficas (SIGs) e modelos de distribuição de espécies (MDEs), associado ao aumento significativo dos registros de ocorrência de espécies em bancos de dados on-line (Sousa-Baena et al. 2014), vem possibilitando avaliações pioneiras no contexto da biologia da conservação (Kamino et al. 2011; Myers et al. 2000). No Brasil, recentemente um estudo utilizando essas ferramentas demonstrou a vulnerabilidade da atual rede de UCs do país, a ponto de menos de $30 \%$ das espécies terem sua distribuição geográfica contempladas por UCs (Oliveira et al.2017). Abordagens conservacionistas têm utilizado essas ferramentas na avaliação da adequação ambiental das espécies (Anderson \& Martínez-Meyer 2004) para a identificação de áreas-chave na definição de prioridades de conservação (Myers et al. 2000; Thorn et al. 2009).

Pleurophora pulchra J.A. Siqueira, V.M. Cotarelli, J.F.B. Pastore \& T.B. Cavalcanti é uma espécie endêmica do DFC e considerada pelos autores como vulnerável de extinção, baseado nos critérios da IUCN (Siqueira-Filho et al. 2015). A distribuição geográfica de $P$. pulchra é pouco conhecida e não nos permite caracterizar ambientalmente os locais adequados ao desenvolvimento e reprodução de suas populações (Siqueira-Filho et al. 2015).

Devido a sua recente descrição em 2015, $P$. pulchra não foi utilizada na primeira avaliação de APCFC (Giulietti et al. 2003), embora se enquadre nos critérios utilizados para tais avaliações. Diante do exposto, o desenvolvimento de estudos biogeográficos e conservacionistas de espécies raras e/ou endêmicas, como P. pulchra, se tornam uma questão multifacetada, especialmente quando há lacunas no conhecimento da distribuição da espécie e as pressões antropogênicas se convergem (Sohn et al. 2013).

Assim, os objetivos do nosso estudo foram modelar a distribuição potencial de $P$. pulchra para o DFC, proporcionando a ampliação do conhecimento biogeográfico da espécie; e investigar sobre as suas exigências ecologicas atráves da caracterização dos locais de elevada probabilidade de ocorrência. Os resultados deste estudo foram confrontados com a atual rede de UCs e APCFCs do DFC visando fomentar discussões acerca da definição/ delineamento de áreas de proteção na Caatinga.

\section{Material e métodos}

\section{Caracterização da espécie}

O gênero Pleurophora pertence à família Lythraceae, sendo exclusivo da América do Sul (Graham et al. 2006). Até o presente momento seis espécies são aceitas para o gênero, ocorrendo na Argentina, Bolivia, Brasil, Chile e Paraguay (Graham et al. 2006). Para o Brasil, atualmente são conhecidas três espécies, $P$. anomala (A. St.-Hil.) Koehne, P. pulchra J.A. Siqueira, V.M. Cotarelli, J.F.B. Pastore \& T.B. Cavalcanti e P. saccocarpa Koehne, sendo que as duas primeiras ocorrem na Caatinga, $P$. anomala em margens de áreas alagadas (rios, ricachos, açudes e etc.) e $P$. pulchra em afloramentos rochosos em serras (Siqueira-Filho et al. 2015). A distribuição geográfica de $P$. pulchra é conhecida apenas para o estado de Pernambuco (Siqueira-Filho et al. 2015; BFG 2018). P. saccocarpa ocorre no estado de Mato Grosso do Sul em áreas alagadas na região do Pantanal, e no Paraguai na região do chaco (Siqueira-Filho et al. 2015).

Na descrição original da espécie, SiqueiraFilho et al. (2015) descrevem de maneira simples sua biologia floral, com destaque para maturação assincrônica entre os estames e estilete, apresentando protrandria, fato que dificulta a autopolinização. De acordo com observações de campo e as características florais da espécie, como coloração vermelha e formato do tubo floral (Fig. 1), é possível que o beija-flor seja um de seus polinizadores. A dispersão das sementes é anemocórica, os frutos são secos e as sementes permanecem no cálice floral no corpo da planta até secarem e se desprenderem (observações pessoais).

A espécie apresenta floração em um período de seis meses durante um ano, de abril a setembro, e frutos de junho a setembro (Siqueira-Filho et al. 2015). 

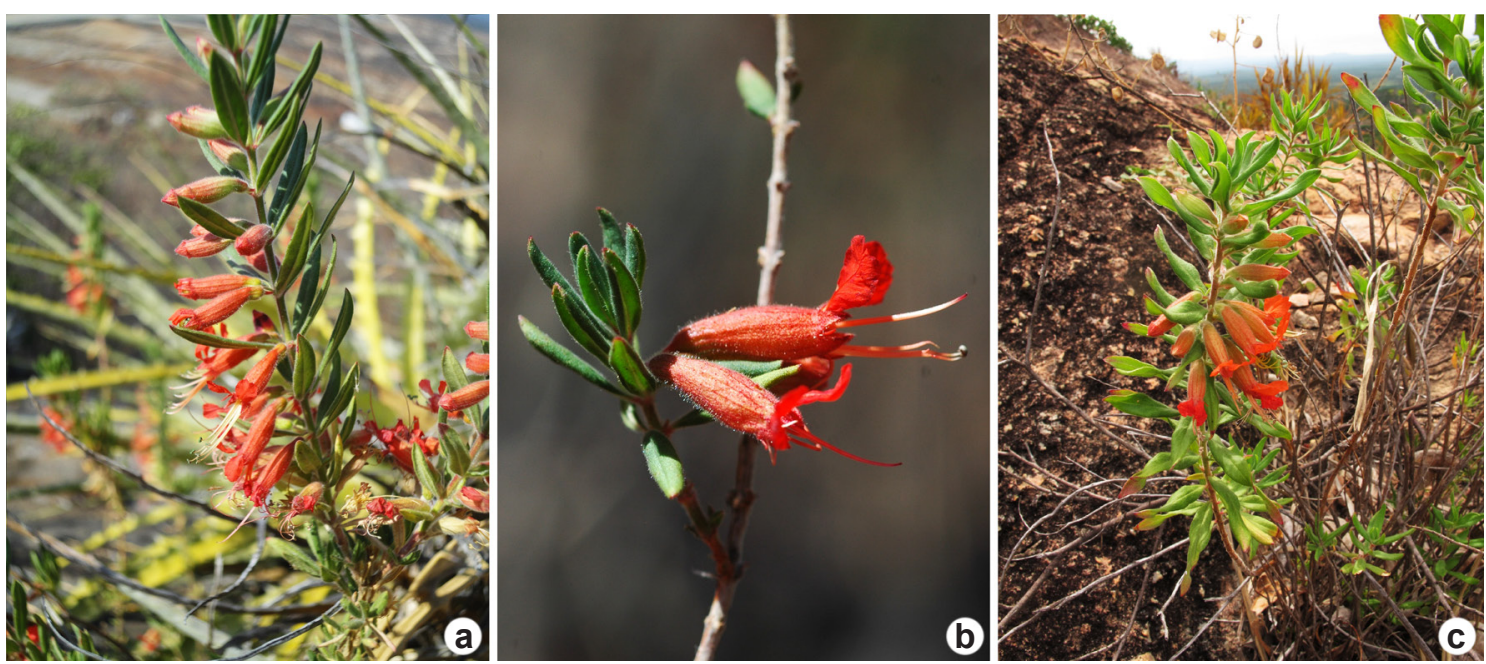

Figura 1 - Pleurophora pulchra (Lythraceae) - a. ramo florido; b. detalhe da flor; c. espécie em ambiente rupícola. (a,b. V.M. Cotarelli; c. A.P. Fontana)

Figure 1 - Pleurophora pulchra (Lythraceae) - a. flowery branch; b. flower detail; c. species in rupicolous environment. (a,b. V.M. Cotarelli; c. A.P. Fontana)

Em geral os locais onde as plantas foram amostradas são caracterizados por serem de serras com altitudes entre 450 e $650 \mathrm{~m}$ (Siqueira-Filho et al. 2015), sempre em afloramentos rochosos, desde áreas mais abertas com incidência solar direta, até um pouco sombreadas. Destaca-se a ocorrência em locais com pouco ou nenhum substrato até em fendas das rochas (observação pessoal).

\section{Coleta e análise dos dados}

Os registros de Pleurophora pulchra foram extraídos da rede virtual de herbários brasileiros (ver Herbário da Universidade Estadual de Feira de Santana (HUEFS) \& Herbário Vale do São Francisco (HVASF). Todos os registros de $P$. pulchra são provenientes exclusivamente de avaliações do material coletado e depositado nos herbários citados e da descrição original da espécie, tornando as determinações e pontos de coleta de alta confiabilidade.

Ao sobrepormos os 11 registros conhecidos da espécie sobre as células da grade (pixels) das variáveis, nós observamos que alguns desses estiveram no mesmo pixel, o que torna os registros autocorrelacionados. Essa sobreposição de pontos não atende a premissa de pontos independentes para construção de SDM (Phillips et al. 2006; Pearson et al. 2007). Assim, nós selecionamos cinco dos 11 registros conhecidos da espécie, para uso no processo de modelagem (Tab. 1).

Optamos pelo uso do algoritmo Maxent (Phillips et al. 2006) na construção dos nossos modelos. O Maxent tem se mostrado eficiente com bons índices de acurácia na aproximação da distribuição geográfica de espécies com distribuição restrita ou ocorrências conhecidas

Tabela 1 - Registros de Pleurophora pulchra utilizados no MDEf.

Table 1 - Records of Pleurophora pulchra used in MDEf.

\begin{tabular}{ccccc}
\hline \multirow{2}{*}{ Registro } & Herbário & \multicolumn{2}{c}{ Coordenadas em graus decimais } & Município/Estado \\
& & Longitude & Latitude & Cabrobó - PE \\
1 & HVASF/CEN/HUEFS & $-39,1875$ & $-8,415278$ & Cabrobó - PE \\
2 & HVASF/RB & $-39,381111$ & $-8,341389$ & Cabrobó - PE \\
3 & IPA & $-39,322778$ & $-8,226389$ & Salgueiro - PE \\
4 & HVASF & $-39,294722$ & $-8,218611$ & Serrita - PE \\
\hline
\end{tabular}


limitadas a registros superior ou igual a cinco (Hernandez et al. 2006; Pearson et al. 2007; Proosdij et al. 2016; Thorn et al. 2009).

Reunimos um conjunto de 24 camadas ambientais com resolução espacial de $1 \mathrm{~km}^{2}$ (Tab. 2) que refletissem vários aspectos edáficos e ou topográficos, de temperatura, precipitação e sazonalidade que possivelmente seriam importantes na determinação da distribuição de $P$. pulchra na caatinga. No entanto, selecionamos algumas variáveis ambientais através de Análise de Componentes Principais (PCA) para minimizar a multicolinearidade e evitar a parametrização excessiva do nosso modelo com variáveis redundantes (Moura-Júnior et al. 2016; Dormann et al. 2013; Mellin et al. 2010; Peterson et al. 2007). Esta análise tem sido indicada para seleção de variáveis, por ser robusta e detectar relações complexas entre as variáveis (Eisenlohr 2014). Assim, selecionamos os 4 primeiros eixos da PCA que juntos explicaram $>85 \%$ da variação geral dos parâmetros ambientais. Selecionamos a variável ambiental que apresentou maior autovalor de cada eixo da PCA (Tab. 3). As variáveis ambientais selecionadas pela PCA foram: Temperatura média do trimestre mais frio (bio11), Precipitação anual (bio12), Sazonalidade de precipitação (bio15), índice de aridez (ia). Incluímos a variável solo no processo

Tabela 2 - Nomenclatura, abreviação, plataforma de download e fonte das camadas ambientais utilizadas na construção da MDE.

Table 2 - Nomenclature, abbreviation, download site and source of the bioclimatic layers used in models computation.

\begin{tabular}{|c|c|c|c|}
\hline Abreviação & Variáveis & Plataformas & Fonte \\
\hline bio1 & Temperatura média anual & Worldclim & Hijmans et al. 2005 \\
\hline bio2 & Amplitude diurna média mensal & Worldclim & Hijmans et al. 2005 \\
\hline bio3 & Isotermalidade (bio2/bio7) $(* 100)$ & Worldclim & Hijmans et al. 2005 \\
\hline bio4 & Sazonalidade da temperatura $=($ desvio padrão $* 100)$ & Worldclim & Hijmans et al. 2005 \\
\hline bio5 & Temperatura máxima do mês mais quente & Worldclim & Hijmans et al. 2005 \\
\hline bio6 & Temperatura mínima do mês mais frio & Worldclim & Hijmans et al. 2005 \\
\hline bio7 & Intervalo da temperatura anual (bio5-bio6) & Worldclim & Hijmans et al. 2005 \\
\hline bio8 & Temperatura média do trimestre mais úmido & Worldclim & Hijmans et al. 2005 \\
\hline bio9 & Temperatura média do trimestre mais seco & Worldclim & Hijmans et al. 2005 \\
\hline bio10 & Temperatura média do trimestre mais quente & Worldclim & Hijmans et al. 2005 \\
\hline bio11 & Temperatura média do trimestre mais frio & Worldclim & Hijmans et al. 2005 \\
\hline bio12 & Precipitação anual & Worldclim & Hijmans et al. 2005 \\
\hline bio13 & Precipitação do mês mais chuvoso & Worldclim & Hijmans et al. 2005 \\
\hline bio14 & Precipitação do mês mais seco & Worldclim & Hijmans et al. 2005 \\
\hline bio15 & Sazonalidade de precipitação (coeficiente de variação) & Worldclim & Hijmans et al. 2005 \\
\hline bio16 & Precipitação do trimestre mais úmido & Worldclim & Hijmans et al. 2005 \\
\hline bio17 & Precipitação do trimestre mais seco & Worldclim & Hijmans et al. 2005 \\
\hline bio18 & Precipitação do trimestre mais quente & Worldclim & Hijmans et al. 2005 \\
\hline bio19 & Precipitação do trimestre mais frio & Worldclim & Hijmans et al. 2005 \\
\hline pet & Evapotranspiração & CGIAR-CSI & Zomer et al. 2007, 2008 \\
\hline ia & Índice de Aridez & CGIAR-CSI & Zomer et al. 2007, 2008 \\
\hline solos & Solo & AMBDATA & Embrapa 1999 \\
\hline alt & Altitude & AMBDATA & Valeriano 2008 \\
\hline decliv & Declividade & AMBDATA & Valeriano 2008 \\
\hline
\end{tabular}


de modelagem por considera-la fundamental no entendimento da distribuição da espécie. Para computar a PCA utilizamos o software R (R CORE 2016), pacote Vegan.

Após a seleção das camadas preditoras pela PCA, computamos cinco modelos independentes excluindo um dos cinco registros de ocorrência da espécie em cada modelo para ser utilizado como ponto teste. Caso tivéssemos sorteados e separados os pontos teste e treino pelo Maxent correríamos o risco de todas as otimizações serem realizadas pelos mesmos pontos treino, bem como que modelo fosse testado pelo mesmo ponto teste. Esse viés é comum em SDM de espécies com poucos registros de ocorrência (Pearson et al. 2007). Nesse caso, recomenda-se que a SDM da espécie seja testada partir da computação de modelos independentes, maximizando-se o número de combinações possíveis de pontos treino e teste (Pearson et al. 2007).

Para tanto, é recomendado que avaliação do desempenho dos modelos independentes seja realizado por teste binomial (Pearson et al. 2007). O teste binomial se baseia em duas proporções: $1^{\circ}$ na proporção da área indicada em cada modelo independente em relação ao background (Domínio Fitogeográfico da Caatinga); $2^{\circ}$ na proporção entre o número de pontos teste previstos nos modelos independentes frente ao número de registros da espécie (Phillips et al. 2004, 2006). O teste binomial foi realizado no software BioEstat 5.0 (Ayres et al. 2007). Por fim, construímos um modelo final otimizando os cinco registros de ocorrência de $P$. pulchra com as camadas preditoras selecionadas pela PCA.

Tabela 3 - Autovalor das variáveis ambientais para os quatro eixos significativos da PCA. As variáveis selecionadas para cada eixo significativo da PCA estão marcadas em negrito.

Table 3 - Eigenvalue of environmental variable for the four significant axes of PCA. The variables selected by significant axis of the PCA are marked in bold.

\begin{tabular}{ccccc}
\hline Variáveis & Eixo 1 & Eixo 2 & Eixo 3 & Eixo 4 \\
\hline ai & 0.0913 & $\mathbf{0 . 3 6 2 6}$ & 0.3064 & 0.1169 \\
alt & 0.2177 & 0.1293 & 0.3108 & 0.1335 \\
bio1 & 0.2898 & 0.0384 & 0.1468 & 0.0998 \\
bio10 & 0.2777 & 0.029 & 0.1875 & 0.154 \\
bio11 & $\mathbf{0 . 2 9 9 6}$ & 0.0212 & 0.0682 & 0.0415 \\
bio12 & 0.1496 & 0.283 & $\mathbf{0 . 3 3 3 2}$ & 0.1924 \\
bio13 & 0.2164 & 0.2046 & 0.2966 & 0.0476 \\
bio14 & 0.1602 & 0.2966 & 0.1224 & 0.3079 \\
bio15 & 0.21 & 0.1133 & 0.1575 & $\mathbf{0 . 3 8 8 6}$ \\
bio16 & 0.2102 & 0.2128 & 0.3219 & 0.0247 \\
bio17 & 0.1583 & 0.2934 & 0.1268 & 0.3346 \\
bio18 & 0.2061 & 0.0961 & 0.1149 & 0.1588 \\
bio19 & 0.1205 & 0.3172 & 0.0773 & 0.1355 \\
bio2 & 0.0508 & 0.3488 & 0.2846 & 0.2958 \\
bio3 & 0.2093 & 0.1071 & 0.0976 & 0.1842 \\
bio4 & 0.2327 & 0.0032 & 0.1929 & 0.2184 \\
bio5 & 0.2651 & 0.1397 & 0.0378 & 0.2566 \\
bio6 & 0.2619 & 0.1309 & 0.2456 & 0.074 \\
bio7 & 0.0312 & 0.3235 & 0.2676 & 0.3838 \\
bio8 & 0.2511 & 0.138 & 0.1922 & 0.0983 \\
bio9 & 0.2816 & 0.0813 & 0.163 & 0.0401 \\
decliv & 0.0716 & 0.0864 & 0.1765 & 0.12 \\
pet & 0.2008 & 0.2784 & 0.1213 & 0.2802 \\
\hline
\end{tabular}




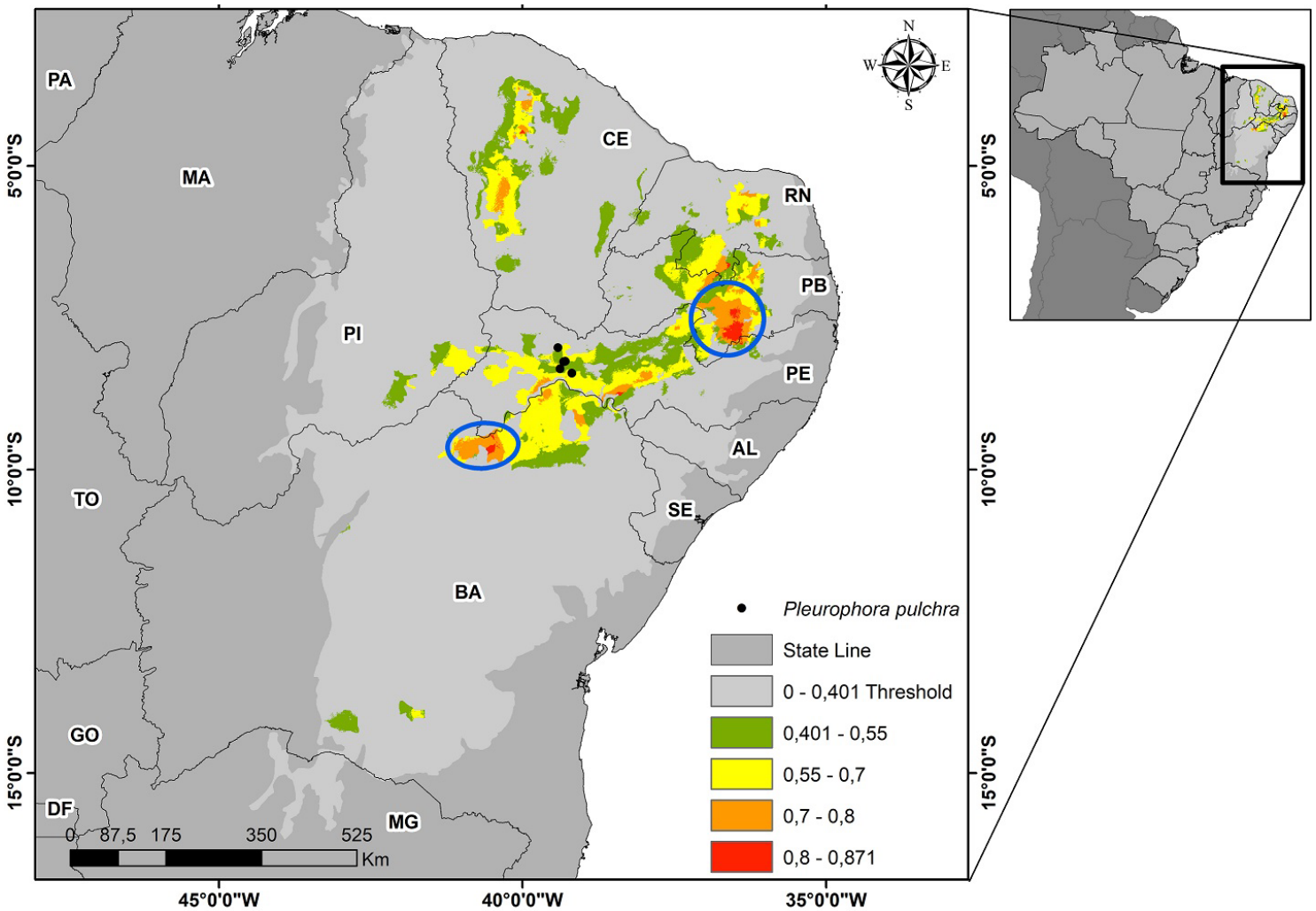

Figura 2 - MDEf para Pleurophora pulchra pelos limites estaduais. Círculos em azul apontam manchas com adequabilidade ambiental superior a 0.7 .

Figure 2 - MDEf for Pleurophora pulchra by state boundaries. Blue circle indicate spots with environmental adequacy greater than 0.7 .

Definimos o limite de corte (Threshold) para distinguir os locais ambientalmente adequados dos não adequados à ocorrência da espécie nos modelos independentes e final através do "limite mínimo de presença” (Pearson et al. 2007).

Para avaliarmos o quanto de área ambientalmente adequada à ocorrência de $P$. pulchra está em áreas conservadas, sobrepomos o MDEf aos shapes de UCs, APCFCs e ecorregiões. Esses shapes foram baseados na classificação de Velloso et al. (2002) para ecorregiões e Ministério do Meio Ambiente - MMA (2016a,b) para UCs e APCFC. Para elaboração dos mapas temáticos e cálculo de proporção de áreas utilizamos o Sistema de Informação Geográficas (SIG) do software ArcGis versão 10.2.

\section{Resultados}

As curvas de resposta das variáveis preditoras evidenciaram que $P$. pulchra ocorre em ambientes de elevada aridez (ia), com baixa precipitação anual (bio12), grande sazonalidade na precipitação (bio15) e temperaturas estáveis nos meses mais frios (bio11) (Apêndice 1, disponibilizado no link $<$ https://doi.org/10.6084/m9.figshare.8184026. v1 $>$ ). Os solos mais ajustados à ocorrência potencial de $P$. pulchra são caracterizados como pouco espessos, variando de imperfeitamente a bem drenados, jovens, pobres em matéria orgânica e/ ou provenientes de poucas alterações em relação ao material de origem (Luvissolo, Planossolos e Neossolos correspondendo a $67.34 \%, 21.21 \%$ e $9.45 \%$ da área predita pelo MDEf, respectivamente).

A avaliação de desempenho pelos modelos independentes evidenciou que $80 \%$ dos registros utilizados como pontos teste estiveram dentro da área ambientalmente adequada a ocorrencia da espécie. O MDEf de $P$. pulchra apresentou desempenho diferente do acaso, segundo o teste binomial $(p<0.001)$ e excelente de acordo com a AUC (0.925) (Pearce \& Ferrier, 2000). A área ambientalmente adequada à ocorrência da espécie no modelo foi de $114.100 \mathrm{~km}^{2}$, o equivalente a $13.79 \%$ do Background (Fig. 2). 
A distribuição potencial de P. pulchra pelo MDEf indicou áreas contínuas que formam um arco entre a região central do Rio Grande do Norte até as regiões centrais da Paraíba e Pernambuco (sertão), Norte da Bahia e Sudeste do estado do Piauí. Além de áreas no Ceará e Sul da Bahia. Dos estados pertencentes à região Nordeste do Brasil, apenas o Maranhão, Alagoas e Sergipe não apresentaram áreas ambientalmente adequadas pelo MDEf. Locais com adequabilidade ambiental superior a 0.7 apontam uma grande área na região do cariri Paraibana e Norte da Bahia com aproximadamente 5.000 e $3.000 \mathrm{~km}^{2}$, respectivamente (círculos em azul na Figura 2).

As áreas ambientalmente adequadas à ocorrência de $P$. pulchra contemplaram predominantemente as ecorregiões da depressão sertaneja meridional e setentrional, com $47.93 \%$ e $37.41 \%$ da área total predita pelo MDEf. Entretanto, foi a ecorregião Planalto da Borborema que se destacou, por alocar maior parte da área com adequabilidade superior a 0.7 do estado da Paraíba (Fig. 2). Estas regiões podem ser caracterizadas como Caatinga sensu strito, com baixa precipitação e altas temperaturas.

Da atual rede de UCs da Caatinga, 15 unidades tiveram áreas preditas pelo MDEf, representando apenas $1.3 \%$ da área total do modelo (Fig. 3). Dessas UCs, as Áreas de Proteção Ambiental (APA) do Cariri e Lago de Sobradinho destacaram-se por contemplar áreas com adequabilidade ambiental acima de 0.7 para a ocorrência de $P$. pulchra. No entanto, a APA Lago de Sobradinho apresentou menos de $4 \%$ de sua extensão como ambientalmente adequadas à ocorrência de $P$. pulchra.

Da área total predita pelo MDEf, 30.59\% sobrepuseram APCFCs, percentagem essa que corresponde a 82 APCFCs. Dessas APCFCs, 23 apresentam adequabilidade ambiental acima de 0.7 para a ocorrência de P. pulchra, sendo que, mais de dois terços dessas, estão classificadas na prioridade para conservação muito alta a extremamente alta. Entretanto, somente a APCFC Jatobá e APCFC Cariri Paraibano (Fig. 4), apresentaram mais que $50 \%$ de suas áreas adequada pelo MDEf. Ambas áreas prioritárias destacadas acima, estão situadas na mesorregião da Borborema no estado da Paraíba.

\section{Discussão}

A ocorrência de P. pulchra em ambientes com elevada temperatura e em afloramentos rochosos já havia sido mencionado na descrição original da espécie (Siqueira-Filho et al. 2015).
As correlações apontadas entre as variáveis ambientais e a ocorrência de $P$. pulchra no MDEf concordam, de forma geral, com a descrição dos locais de coleta, sugerindo a eficácia do modelo produzido. Entretanto, era desconhecido, até o momento, que $P$. pulchra ajustava-se em ambientes de baixa precipitação, elevada sazonalidade de precipitação, temperaturas estáveis nos meses de maior precipitação, solos rasos ou pouco profundos, medianamente drenados e com baixos teores de matéria orgânica.

A distribuição geográfica de $P$. pulchra é conhecida apenas para o estado de Pernambuco (Siqueira-Filho et al. 2015; BFG 2018). Entretanto, o mapa obtido pelo SDMf aponta extensas áreas ambientalmente adequadas a sobrevivência da espécie na região central Paraibana e norte da Bahia. Além disso, esse modelo indicou áreas que formam um arco entre a região central do Rio Grande do Norte até o Sudeste do Piauí, enfatizando assim a necessidades de futuras expedições exploratórias em diversos ambientes localizados nesses estados para verificação da ocorrência da espécie e ou reavaliação de material coletado que pode ter sido identificado de maneira equivocada.

É possível que exsicatas de P. pulchra depositados em herbários do Brasil possam ter identificação trocada por espécies do gênero Cuphea, indicado como grupo irmão de Pleurophora (Graham et al. 2006). Cuphea possui características florais similares com Pleurophora, como por exemplo, cálice conspícuo, que em algumas espécies pode apresentar coloração vermelha (ex. C. pulchra Moric). É possível também que haja erros de identificação nas exsicatas de Pleurophora anomala (A. St.-Hil.) Koehne, a qual era considerada até 2015 a única espécie desse gênero reconhecida para o DFC. As áreas indicadas pelo MDEf como ambientalmente adequadas a ocorrência de $P$. pulchra são coincidentes com a distribuição de $P$. anomala e vários géneros de Cuphea. Isso sustenta a possibilidade de erros de identificação das exsicatas de Pleurophora e/ ou Cuphea depositadas em herbários brasileiros e evidenciam a necessidade da avaliação por taxonomistas, especialistas de Lythraceae, nessas exsicatas.

Embora a MDEf tenha manchado predominantemente áreas da depressão sertaneja meridional (onde encontram-se as populações de P. pulchra) e setentrional, áreas da ecorregião do planalto da Borborema Paraibano também apresentou áreas com elevada adequabilidade 


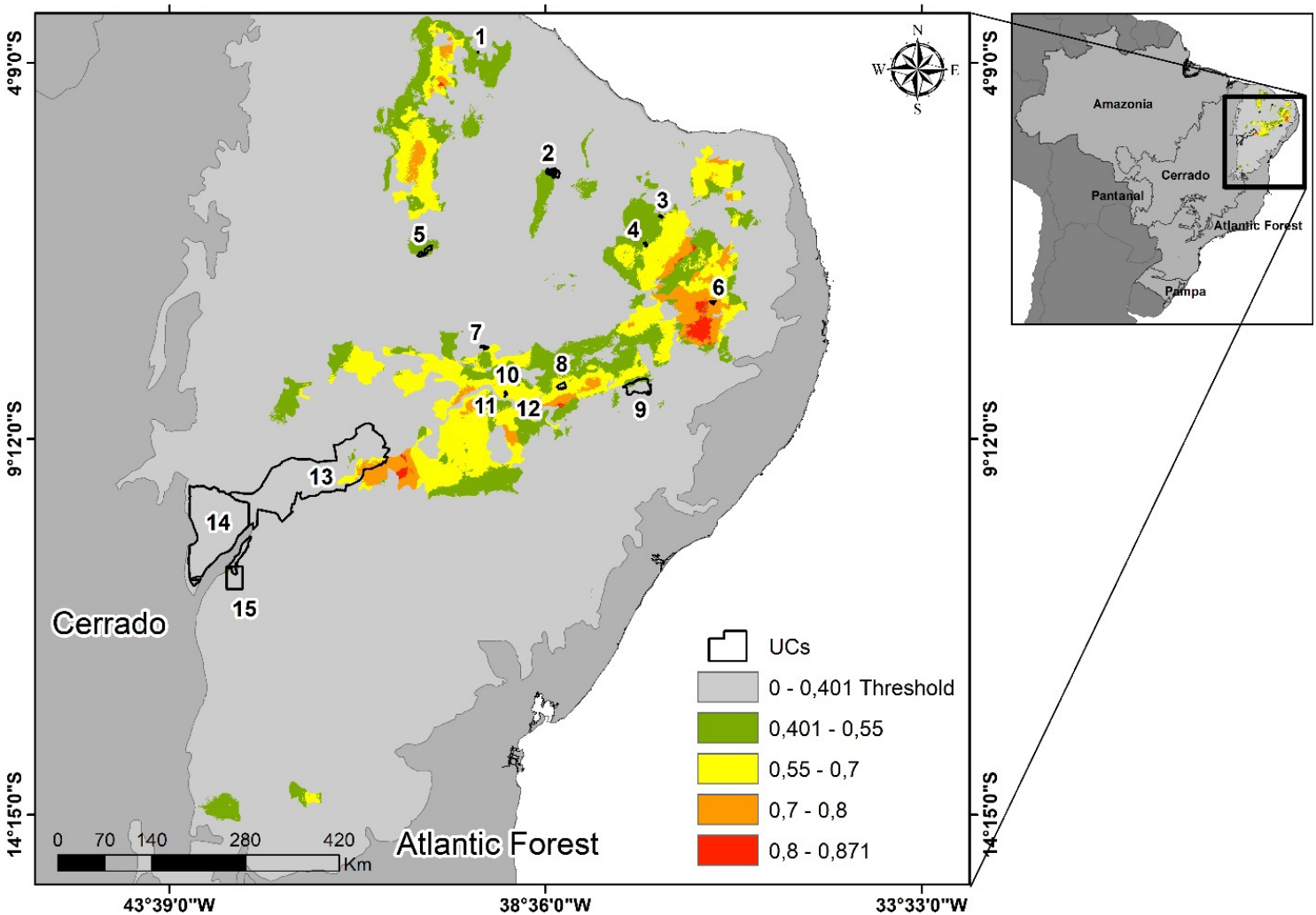

Figura 3 - MDEf para Pleurophora pulchra pelas UCs inseridas no Domínio Caatinga com adequabilidade ambiental acima de 0.70. 1 - RPPN Elias Andrade; 2 - Estação Ecológica do Castanhão; 3 - RPPN Fazenda Salobro; 4 - Estação Ecológica do Seridó; 5 - Estação Ecológica de Aiuaba; 6 - APA do Cariri; 7 - Floresta Nacional de Negreiros; 8 Estação Ecológica Serra da Canoa; 9 - Parque Nacional do Catimbau; 10 - RPPN Reserva Jurema; 11 - RPPN Reserva Umburana; 12 - RPPN Reserva Siriema; 13 - APA Lago De Sobradinho; 14 - APA Dunas e Veredas do Baixo Médio São Francisco; 15 - APA Lagoa De Itaparica.

Figure 3 - SDMf for Pleurophora pulchra by Conservation Units within Caatinga Dominium with environmental adequacy greater than 0.7. 1 - RPPN Elias Andrade; 2 - Estação Ecológica do Castanhão; 3 - RPPN Fazenda Salobro; 4 - Estação Ecológica do Seridó; 5 - Estação Ecológica de Aiuaba; 6 - APA do Cariri; 7 - Floresta Nacional de Negreiros; 8 - Estação Ecológica Serra da Canoa; 9 - Parque Nacional do Catimbau; 10 - RPPN Reserva Jurema; 11 - RPPN Reserva Umburana; 12 - RPPN Reserva Siriema; 13 - APA Lago De Sobradinho; 14 - APA Dunas e Veredas do Baixo Médio São Francisco; 15 - APA Lagoa De Itaparica.

ambiental para a espécie. O resultado do teste binomial evidenciou que os procedimentos de otimização dos modelos independentes foram capazes de definir bons preditores ambientais e, consequentemente, áreas adequadas à ocorrência de P. pulchra no Background (Phillips et al. 2004, 2006). Isso reforça a possibilidade de novos registros para a espécie nas áreas supracitadas no MDEf.

A presença confirmada de populações de $P$. pulchra em UC de proteção integral (Siqueira-Filho et al. 2015) demonstra a importância desse tipo de unidade para manutenção da espécie em ambientes naturais. O status "vulnerável" para ameaça de extinção de $P$. pulchra e as projeções de diminuição da qualidade de seu habitat (Siqueira-Filho et al. 2015) ratificam a importância das 2 UCs e 23 APCFCs com elevada adequabilidade ambiental $(>0.7)$ para a espécie no MDEf, com destaque para as APCFCs Jatobá e Cariri Paraibano. Por isso, sugerimos um esforço de coleta direcionado nessas UCs e APCFCs.

Os estudos de MDE são vistos como fundamentais nas etapas iniciais do processo de seleção de áreas com alto valor para a conservação da biodiversidade (Myers et al. 2000; Koch et al. 2017). Por isso, os resultados do nosso estudo poderão subsidiar também futuras discussões sistêmicas acerca de seleção de áreas importantes para conservação da biodiversidade ou valoração 

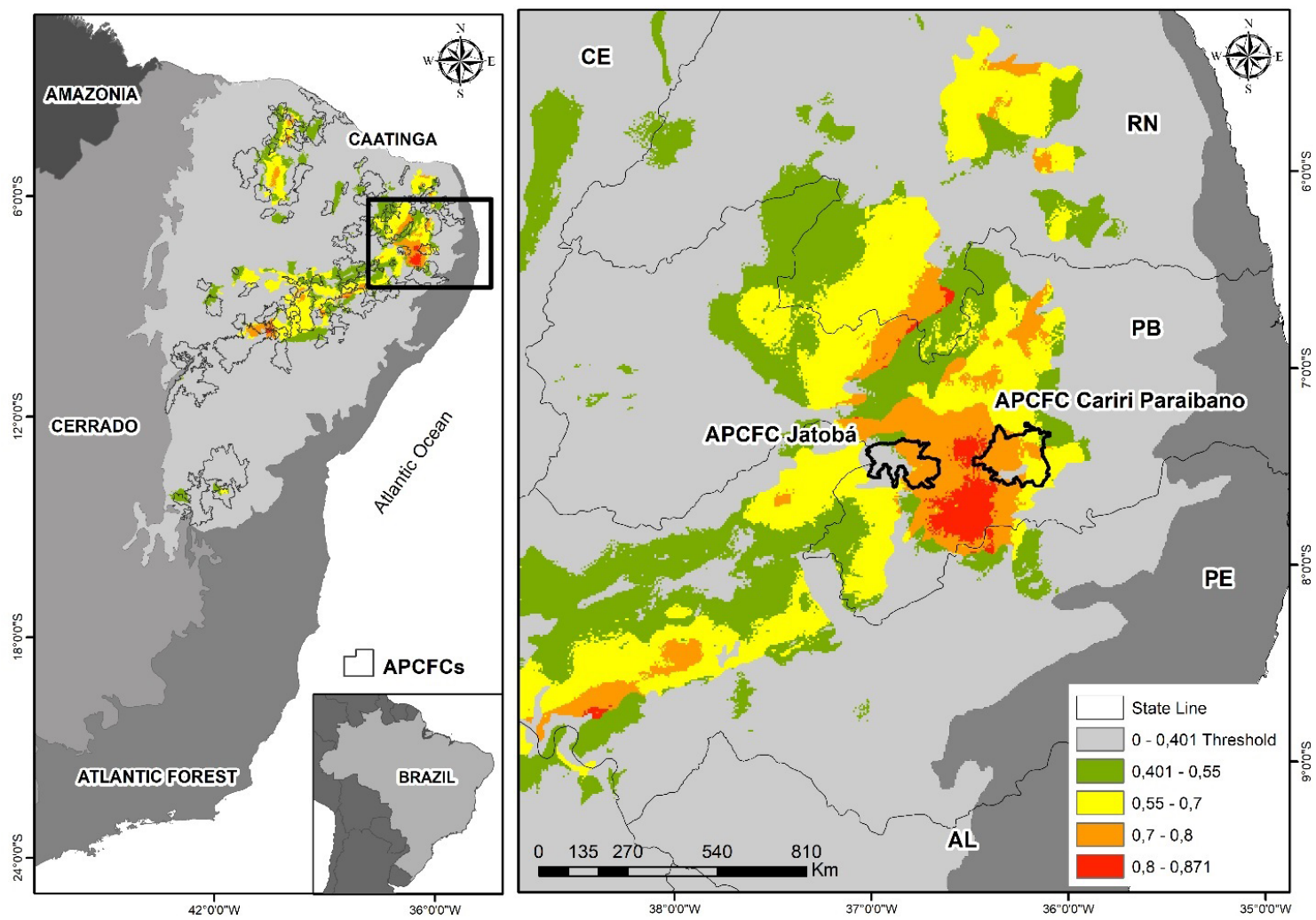

Figura 4 - SDMf para Pleurophora pulchra pelas APCFCs que apresentaram mais de 50\% de suas áreas com adequabilidade ambiental acima de 0.70 .

Figure 4 - SDMf for Pleurophora pulchra by APCFCs that exhibit more than $50 \%$ of environmental adequacy greater than 0.7 .

das UC e APCFCs já existentes no DFC. Nesse contexto, ressaltamos as UCs APAs do Cariri e Lago de Sobradinho que permitem o uso sustentável de seus recursos, além das APCFC Jatobá e APCFC Cariri Paraibano pela possibilidade de elevação do grau de prioridade de conservação, que atualmente é extremamente alta.

Em síntese, constatamos que $P$. pulchra está potencialmente relacionada a ambientes de solos rasos, mal drenados e pobres em matéria orgânica com condições climáticas de elevada aridez e sazonalidade de precipitação, baixa precipitação e temperaturas estáveis nos meses de maior precipitação. As áreas indicadas pelo MDEf indicaram predominantemente a depressão sertaneja meridional e setentrional, além de áreas do planalto da Borborema da Paraíba. Das UCs e APCFCs somente as unidades/áreas situadas na mesorregião da Borborema no estado da Paraíba (APA do Cariri; APCFC Jatobá e APCFC Cariri Paraibano) apresentaram mais que $50 \%$ de suas áreas com adequação superior a 0.7. Diante do aprofundamento na caracterização ambiental das áreas relacionadas à potencial ocorrência de $P$. pulchra, esse trabalho apresenta uma contribuição ao conhecimento ecológico e biogeográfico da espécie e discussões para futuras avaliações sobre APCFC e UC.

\section{Referências}

Ayres M, Ayres MJ, Ayres DL \& Santos AAS (2007) BIOESTAT 5.0: aplicações estatísticas nas áreas das ciências biológicas e médicas. Sociedade Civil Mamirauá/CNPq, Brasília. 324p.

BFG - The Brazil Flora Group (2018) Brazilian Flora 2020: innovation and collaboration to meet Target 1 of the Global Strategy for Plant Conservation (GSPC). Rodriguésia 69: 1513-1527.

Castelletti CHM, Silva JMC, Tabarelli M \& Santos AMM (2004) Quanto ainda resta da Caatinga? Uma estimativa preliminar. In: Silva JMC, Tabarelli M, Fonseca MT \& Lins LV (orgs.) Biodiversidade da Caatinga: áreas e ações prioritárias para a conservação. Ministério do Meio Ambiente, Brasília. Pp. 91-100. 
De Moura-Júnior EG, Valentin-de-Souza RL \& Milhomens LBS (2016) New record of Pithophora roettleri (Roth) Wittrock, 1877 (Chlorophyta: Pithophoraceae) for the São Francisco River basin and its potential geographic distribution in Brazil. Check List 12: 1899.

Dormann CF, Elith J, Bacher S, Buchmann C, Carl G, Carré G, Marquéz JRG, Gruber B, Lafourcade B, Leitão PJ, Münkemüller T, McClean C, Osborne PE, Reineking B, Schröder B, Skidmore AK, Zurell D \& Lautenbach S (2013) Collinearity: a review of methods to deal with it and a simulation study evaluating their performance. Ecography 36:27-46.

Eisenlohr PV (2014) Persisting challenges in multiple models: a note on commonly unnoticed issues regarding collinearity and spatial structure of ecological data. Brazilian Journal of Botany 37: 365-371.

Giulietti AM, Bocage Neta ALD \& Paula ARL (2003) Vegetação: áreas e ações prioritárias para a conservação da Caatinga. In: Silva JMC, Tabarelli M, Fonseca MT \& Lins LV. Biodiversidade da caatinga: áreas e ações prioritárias para a conservação. Ministério do Meio Ambiente / Universidade Federal de Pernambuco, Brasília. Pp. 113-132.

Graham SA, Freudenstein JV \& Lucker M (2006) A phylogenetic study of Cuphea (Lythraceae) based on morphology and nuclear rDNA ITS sequences. Systematic Botany 31: 764-778.

Herbário da Universidade Estadual de Feira de Santana (HUEFS), Herbário Vale do São Francisco (HVASF). Disponível em <http://www.splink.org. br>. Acesso em 5 setembro 2016.

Hernandez PA, Graham CH, Master LL \& Albert DL (2006) The effect of sample size and species characteristics on performance of different species distribution modeling methods. Ecography 29: 773-785.

Kamino LHY, Stehmann JR, Amaral S, De Marco Jr P, Rangel TF, de Siqueira MF, De Giovanni R \& Hortal J (2011) Challenges and perspectives for species distribution modeling in the neotropics. Biology Letters 8: 324-326.

Koch R, Almeida-Cortez JS \& Kleinschmit B (2017) Revealing areas of high nature conservation importance in a seasonally dry tropical forest in Brazil: combination of modelled plant diversity hot spots and threat patterns. Journal for Nature Conservation 25: 24- 39.

Mellin C, Bradshaw CJA, Meekan MG \& Caley MJ (2010) Environmental and spatial predictors of species richness and abundance in coral reef fishes. Global Ecology and Biogeography 19: 212-222.

MMA-Ministério do Meio Ambiente (2016a) Resultados da $2^{\mathrm{a}}$ atualização das áreas prioritárias. Disponível em < http://www.mma.gov.br/biodiversidade/ biodiversidade- brasileira/\%C $3 \% \mathrm{~A} 1$ reaspriorit $\%$ C3\%A1rias/item/10724>. Accesso em 4 April 2017.

MMA - Ministério do Meio Ambiente (2016b) Cadastro Nacional de Unidades de Conservação (atualizado). Disponível em <http://mapas.mma.gov.br/i3geo/ datadownload.htm $>$ Accesso em 4 dezembro 2016.

Oliveira U, Soares-Filho BS, Paglia AP, Brescovit AD, Carvalho CJB, Silva DP, Rezende DT, Leite FSF, Batista JAN, Barbosa JPPP, Stehmann JR, Ascher JS, Vasconcelos MF, De Marco P, LöwenbergNeto P, Ferro VG \& Santos AJ (2017) Biodiversity conservation gaps in the Brazilian protected areas. Scientific reports 7: 9141.

Pearce J \& Ferrier S (2000) Evaluating the predictive performance of habitat models developed using logistic regression. Ecological modelling 133: 225-245.

Pearson RG, Pearson RG, Raxworthy CJ, Nakamura M \& Peterson AT (2007) Predicting species' distributions from small numbers of occurrence records: a test case using cryptic geckos in Madagascar. Journal of biogeography 34: 102-117.

Peterson AT, Papes M \& Eaton M (2007) Transferability and model evaluation in ecological niche modeling: a comparison of GARP and Maxent. Ecography 30: 550-560.

Phillips SJ, Anderson RP \& Schapired RE (2006) Maximum entropy modeling of species geographic distributions. Ecological Modelling 190: 231-259.

Phillips SJ, Dudík M \& Schapire RE (2004) A maximum entropy approach to species distribution modeling. In: International Conference on Machine Learning. Proceedings of the $21^{\text {st }}$ International Conference on Machine Learning. ACM Press, New York. Pp. 655-662.

Proosdij AS, Sosef MS, Wieringa JJ \& Raes N (2016) Minimum required number of specimen records to develop accurate species distribution models. Ecography 39: 542-552.

R DEVELOPMENT CORE TEAM - R CORE (2016) [viewed 10 Sebtember 2016]. R: a language and environment for statistical computing. R Foundation for Statistical Computing, Vienna. Available at $<$ http://www.R-project.org/>. Access on 30 março 2017.

Sá IB, Cunha TJF, Teixeira AH de C, Angelotti F \& Drumond MA (2010) Processo de desertificação no Semiárido brasileiro. In: Sá IB \& Silva PCG. Semiárido brasileiro. Embrapa Semi-Árido, Petrolina. Pp. 126-158.

Siqueira-Filho JA, Cotarelli VM, Pastore JFB, Graham SA \& Cavalcanti TB (2015) A remarkable new species of Pleurophora (Lythraceae) from Caatinga of Pernambuco, Brazil. Systematic Botany 40: 185-190. 
Sohn N, Fernandez MH, Papes M \& Anciães M (2013) Ecological niche modeling in practice: flagship species and regional conservation planning. Oecologia Australis 17: 429-440.

Sousa-Baena MS, Garcia LC \& Peterson AT (2014) Completeness of digital accessible knowledge of the plants of Brazil and priorities for survey and inventory. Diversity and Distributions 20: 369-381.

Tabarelli M \& Silva JMC (2002) Áreas e ações prioritárias para a conservação, utilização sustentável e repartição de benefícios da biodiversidade do bioma Caatinga. In: Araújo EL, Moura AN, Sampaio EVSR, Gestinari LMS \& Carneiro JMT
Biodiversidade, conservação e uso sustentável da flora do Brasil. Imprensa Universitária da UFRPE, Recife. Pp. 27-30.

Thorn W, Nijman V, Smith D \& Nekaris KAI (2009) Ecological niche modelling as a technique for assessing threats and setting conservation priorities for Asian slow lorises (Primates: Nycticebus). Diversity and Distributions 15: 289-298.

Velloso AL, Sampaio EVSB \& Pareyn FGC (2002) Ecorregiões: propostas para o bioma Caatinga. PNEA - Associação Plantas do Nordeste, Recife; Instituto de Conservação Ambiental, The Nature Conservancy do Brasil, Brasília. Pp. 76. 